\title{
GENDER DIFFERENCES IN TONIC AND PHASIC ELECTRODERMAL ACTIVITY COMPONENTS
}

\author{
Dindar S. Bari ${ }^{\text {a }}$ \\ ${ }^{a}$ Dept. of Physics, Faculty of Science, University of Zakho, Kurdistan RegionIraq (dindar.bari@uoz.edu.krd)
}

\begin{abstract}
:
Electrodermal activity (EDA) is a wellestablished psychophysiological measurement for research and clinical approaches. Males and females often display different physiological responses to stimuli, which can be detected by EDA rec ordings. Using a new method to measure skin conductance (SC), skin potential (SP) and skin susceptance (SS) simultaneously at the same electrode, these differences were investigated. SC, SP, and SS were recorded from 60 participants during relaxation and stress. It wasfund that both tonic and phasic EDA parameters indicated gender differences. In addition, female displayed greater tonic and phasic EDA parameters (except for skin potential responses (SPRs)) than males under both relaxation and conditions of stimulation (stress). However, these results were not statistically significant $(p>0.05)$. This suggests that it is perhaps important to consider gender or at least note type of gender in EDA researches, but this cannot be generalized to clinical approaches
\end{abstract}

KEYWORDS: Electrodermal activity, EDA, Skin conductance, Skin potential, Skin susceptance, Gender.

\section{INTRIDUCTION}

Electrodermal activity (EDA) is the property of the human body that causes continuous changes in the skin electrical properties in response to sweat secretion. The EDA measurements have been done since the 1880s with the pioneering studies of Fere (1888) and Tarchanoff (1889) (Boucsein, 2012). EDA measurements can be used to derive psychophysiological parameters reflecting sympathetic nervous system activity indirectly through its relati on to sweat gland activity, which directly changes, for example, the electrical conductance through the horny layer of the skin upon sweat duct filling. In addition, EDA is regarded as one of the best -known electrophysiological phenomena widely used in var ious applications such as in psychophysiology, ambulatory monitoring, intensive care and biofeedback among others (Tronstad et al., 2010).

EDA can be differentiated into a tonic (level) component with the slow baseline variations and a phasic (response) component with the fast response variations. The latter is associated with an orienting reflex and appears either in response to a stimulus or without evident stimulation (nonspecific or spontaneous responses)(Boucsein, 2012). EDA recordings can be broadly classified into two categories, namely endosomatic and exosomatic measurements. Endosomatic recordings involve measurement the amount of potential differences originating in the skin itself without using any externakource of current. Exosomatic recordings on the other hand, involve measurement the EDA parameters through applying an external source of either alternating current or direct current (Tronstad et al., 2010).

The human skin consists of several layers and the stratumcorneum is the outermost layer of the skin, consisting mostly of dead skin cells in a thickness of generally $15-20$ layers. This keratinized tissue has a very high ionic impedivity under dry conditions compared to the deeper layers of the skin. The impedivity in this skin layer is strongly reduced when sweat ducts are filled with sweat(Tronstad et al., 2008).
EDA c ould possibly be influenced by various demographic characteristics. One of the demographically determined individual differences which can contribute to differing behavior of the electrodermal system, is gender-differences (Boucsein, 2012). The differences between females and males are investigated for sweating. It is shown females have a greater sweat gland density than males but display more delayed and, in total, less sweating (Edelberg, 1971; Morimoto, 1978). Also (Jorgenson et al., 1988) revealed that the number of active sweat glands, which was considerably lower in female compared to males newborns, reported this difference being reversed in children of mean age 7-8 years and in adults of about 25 years. More active sweat glands for fem ales than males, is also mentioned by (Fowles, 1986). Also studies of genderdifferences with respect to EDA have previously been carried out in some investigations, see for example (Edelberg, 1972; El -Sheikh, 2007; Hare et al., 1971; Ketterer \& Smith, 1977; Kimmel \& Kimmel, 1965; Kopacz \& Smith, 1971; Maltzman et al., 1979; Neufeld \& Davidson, 1974; Purohit, 1966, but not with focus on three EDA parameters (SC, SP and SS) recorded simultaneously on the same skin site.

In this study, author aims to investigate gender differences in EDA level (tonic) and responses (phasic) components to some external stimulus by using a new non -invasive bioimpedance system, which dependent on recording the three EDA (SC, SP, and SS) simultaneously at the same skin site.

\section{MATERIALS AND METHOD}

\subsection{PC-based System}

Tonic and phasic EDA were measured via using a new Pebased system for recording skin admittance (SY) and skin potential (SP) simultaneously at the same electrode on the same skin site. The system was consisted from a small front-end electronic box connected through a National Instruments DAQ cardNI USB to a PC laptop running software written in Lab VIEW similar to the system presented in (Bari et al., 2018; Tronstad et al., 2013). A three-electrode measurement setup was used: one measuring 
electrode (M), one reference electrode (R), and a current-sink electrode $(\mathrm{C})$. The $\mathrm{C}$ and $\mathrm{R}$ electrodes were used for unipolar AC SC measurement and simultaneously $\mathrm{M}$ and $\mathrm{R}$ electrodes were utilized for SP measurement. Finally, the quadrature component of the SY signal was used to find the SS ( Bari et al., 2018; Tronstad et al., 2013).

In the present study exosomatic AC current was used, which produced through a Howland current source. A 200_mV sine wave was generated by the DAQ Card and fed to the Howland circuit. Then through Howland circuit it was converted to a $20 \mathrm{~Hz}$ AC current of about $20 \mu \mathrm{A}$ and delivered to the skin through the M electrode. The DAQ card received the analog signals back from the skin through the front-end electronic box and converted it to the digital form. Then digitized signals were processed by differentiation in the PC LabVIEW, and separated into a DC component for SP and an $\mathrm{AC}$ component for SC from the real part of the SY signal and SS from the imaginary part by means of phasesensitive rectification. The reason for choosing signal frequency $(20 \mathrm{~Hz})$ in this study was because a frequency of $20 \mathrm{~Hz}$ provides a good trade-off between measurement speed and sensitivity for sweat duct activity (Pabst et al., 2017).

\subsection{Electrode type and placement}

The employed electrodes were Kendall Kittycat 1050NPSM $\mathrm{Ag} / \mathrm{AgCl}$ solid gel ECG neonatal electrodes with an active area of $5.05 \mathrm{~cm}^{2}$ ( Pabst et al., 2017). This type of electrode is selected since it is found to be suitable for EDA recordings. In addition, they are pre-wired electrodes that are initially covered with solid adhesive hydrogel which provides firm adhesion while minimizing irritation to delicate skin (Tronstad et al., 2010).

Electrodes were placed on one arm of the participants. The $\mathrm{M}$ electrode was placed on the hypothenar site of the palm, the $\mathrm{R}$ electrode on the apex of the elbow, which is an electrodermally inactive area as recommended (Fowles et al., 1981), and finally the $C$ electrode on the underarm between $\mathrm{M}$ and $\mathrm{R}$ as shown in Figure 1, employing a three-electrode measurement system.

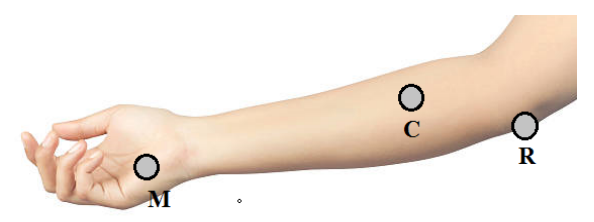

Figure 1. Figure showing electrode placement. The AC current $(20 \mu \mathrm{A})$ provided by the instrumentation is applied to the skin through the $\mathrm{M}$ electrode

\subsection{Participants and Procedure}

Sixty healthy volunteers ( 30 male and 30 females, mean age $36.5 \pm 16$ years, $\mathrm{SD}=16.1$ years) were recruited from the Zakho university staff and students and gave written informed consent.

The experiment took place in a calm indoor environment with normal room temperature $\left(22-23^{\circ} \mathrm{C}\right)$, as recommended (Boucsein, 2012). The participants were seated in a comfortable chair during the experiment ( Bari et al., 2018; Tronstad et al., 2013; Aldosky, 2019). Before recording started, five minutes was allowed for EDA electrode stabilization then EDA were recorded. In order to produce mental stress and EDA responses, the participants were subjected to some external stimuli as illustrated in Table 1 . The mathematical task was subtractions of 13 successively from a starting number of 100 . Before and after stimuli, there was a relaxation time of 60 seconds in order to obtain the baseline of the EDA measurements. Any speaking was not allowed for participants, and they were asked to relax and to avoid bodily movement, during the whole session of EDA recordings.

Table 1. Time schedule of the experiment.

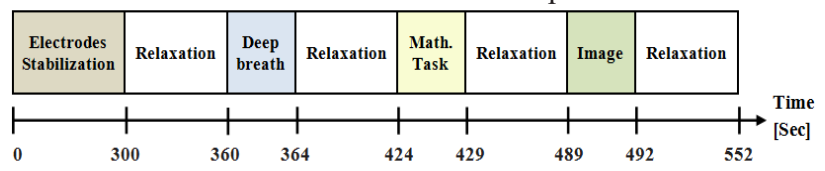

2.4 Data and Statistical Analysis

In order to compare the EDA waveforms recorded from both sexes against each other, several EDA scores (listed in Table 2) were computed from EDA responses for each subject during the task periods. This was done by calculating the mean of onsets and peaks of three responses for all participants. Onsets and peaks were specified analogous to the procedure presented in (Bari et al., 2018).

Table 2. List of scores computed from the EDA responses that were used in the data analyses.

\begin{tabular}{|l|l|l|}
\hline Score & \multicolumn{1}{|c|}{ Definition } & Unit \\
\hline SCRs_Amp & $\begin{array}{l}\text { Amplitude of the skin conductance } \\
\text { response }\end{array}$ & $\mu \mathrm{S}$ \\
\hline SCL & Skin conductance level & $\mu \mathrm{S}$ \\
\hline SPRs_Amp & $\begin{array}{l}\text { Amplitude of the skin potential } \\
\text { response }\end{array}$ & $\mathrm{mV}$ \\
\hline SPL & Skin potential level & $\mathrm{mV}$ \\
\hline SSR_Amp & $\begin{array}{l}\text { Amplitude of the skin susceptance } \\
\text { response }\end{array}$ & $\mu \mathrm{S}$ \\
\hline SSL & Skin susceptance level & $\mu \mathrm{S}$ \\
\hline
\end{tabular}

The EDA waveforms recorded from both sexes were evaluated statistically using all the extracted scores listed in Table 2. For this purpose, the Mann-Whitney U test was employed and the 0.05 level of confidence was used to define statistical significance. The analysis was done using IBM SPSS Statistics 22.

\section{RESULTS}

\subsection{Tonic EDA parameters}

3.1.1 Skin Conductance Level (SCL): Data for SCL over 60 test subjects as a function of gender are presented in Figure 2. Although females showed a greater median SCL value than males, these findings were statistically insignificant $(p>0.05)$.

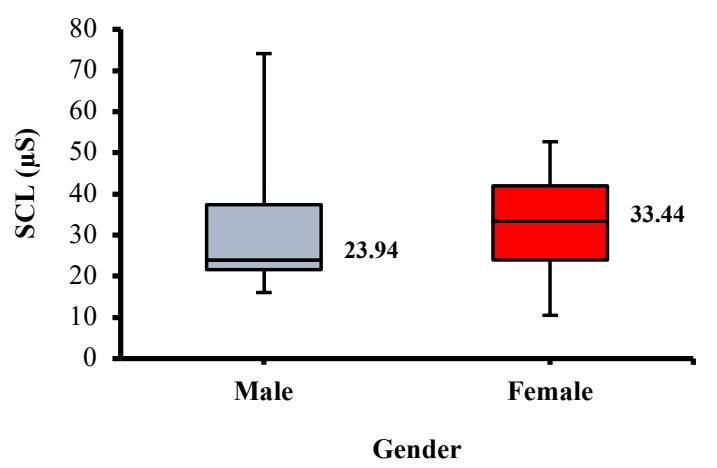

Figure 2. Box-plot with medians, quartiles, and the minimum and maximum as whiskers, shows SCL for all subjects of both genders. 
3.1.2 Skin Potential Level (SPL): Figure 3 shows the box-plot of SPL for all 60 skin samples at each group. It can be seen that the SPL for females is negatively higher than males, as indicated through the median value of the box-plot. Moreover, the statistical analysis with the Mann-Whitney U test also showed the difference between males and females was significant $(p<0.05)$ as noted in the Figure.

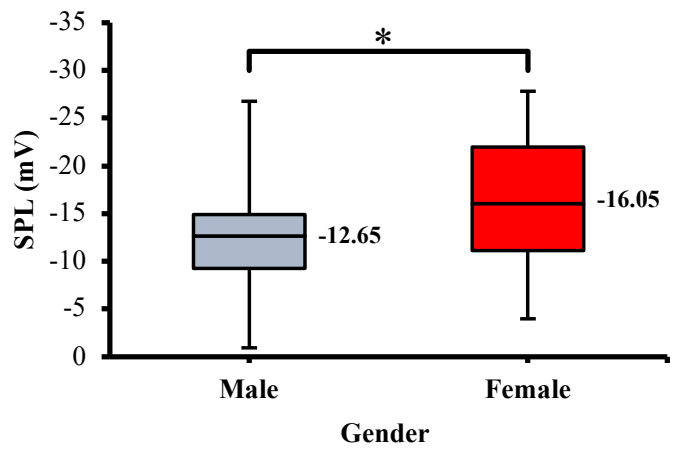

Figure 3. Box-plot with medians, quartiles, and the minimum and maximum as whiskers, shows SPL for all subjects of both genders. ${ }^{*} p<0.05$.

3.1.3 Skin Susceptance Level (SSL): The SSL data for all subjects together are presented with box-plot in Figure 4. The Figure shows that the median value of SSL for males is slightly lower than females, but this result was not supported statistically since $(p>0.05)$.

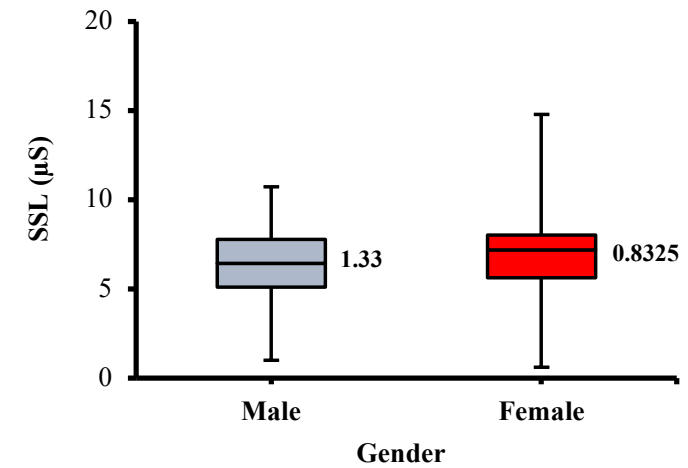

Figure 4. Box-plot with medians, quartiles, and the minimum and maximum as whiskers shows SSL for all subjects of both genders.

\subsection{Phasic EDA parameters (Amplitudes of EDA)}

3.2.1 Skin Conductance Responses (SCRs): Figure 5A, $\mathrm{B} \& \mathrm{C}$ present the median value of SCRs amplitude for different stimuli with respect to both genders. Medians SCRs amplitudes of females group were slightly higher than males individuals. However, when data for SCRs amplitude were statistically analyzed, no significant $(p>0.05)$ difference between both sexes was observed.
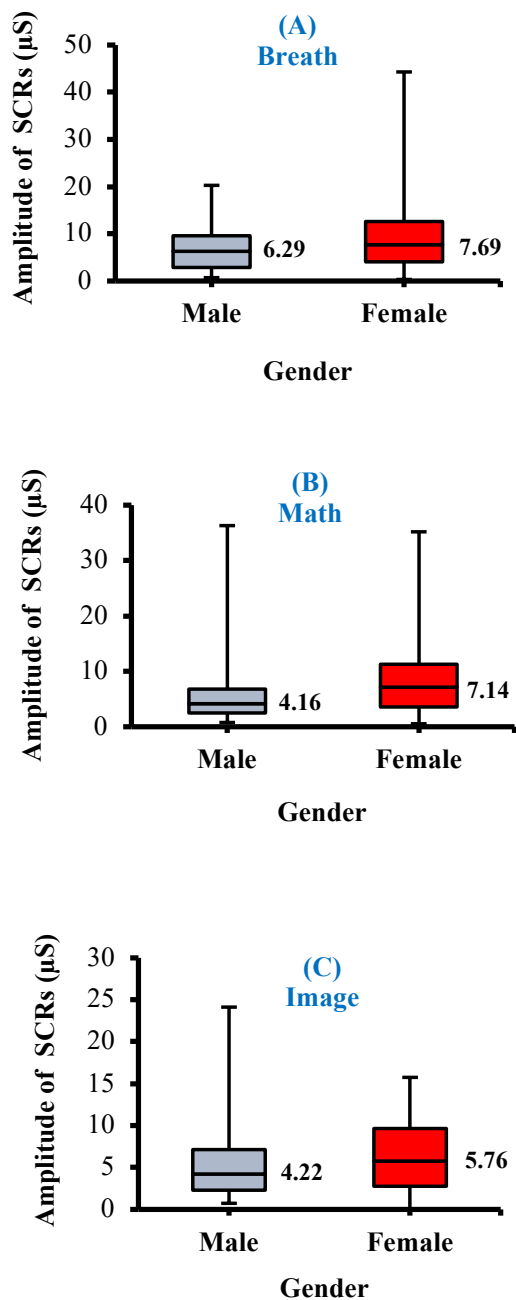

Figure 5A, B\&C. Box-plot with medians, quartiles, and the minimum and maximum as whiskers shows amplitude of SCRs for both genders as a function of deep breath, math, and image stimuli.

3.2.2 Skin Potential Responses (SPRs): The results for SPRs amplitude with respect to the type of stimulus and different genders are shown in Figure 6A, B\&C. As seen response amplitudes for females were lower than males. However, once statistical analyses were performed no significant $(p>0.05)$ difference between data of both groups was obtained as indicated by Mann-Whitney U test.

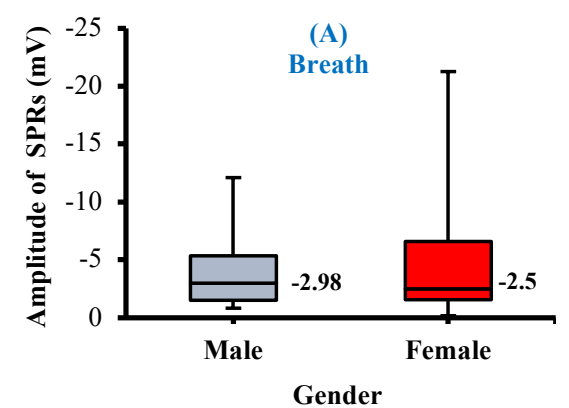



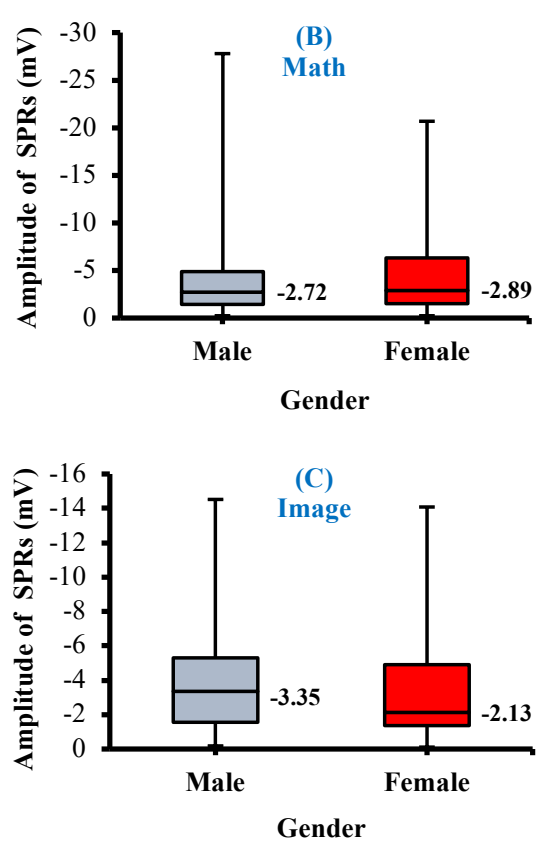

Figure 6 A, B \&C. Box-plot with medians, quartiles, and the minimum and maximum as whiskers shows amplitude of SPRs for both genders as a function of deep breath, math, and image stimuli

3.2.3 Skin Susceptance Responses (SSRs): Amplitudes of SSRs were also obtained for both males and females groups to each stimulus and are presented in Figure 7A, $\mathrm{B} \& \mathrm{C}$. It can be seen that there are differences between the two groups in which females showed higher median amplitudes of SSR than males, but these results were not found to be statistically significant $(p>0.05)$.
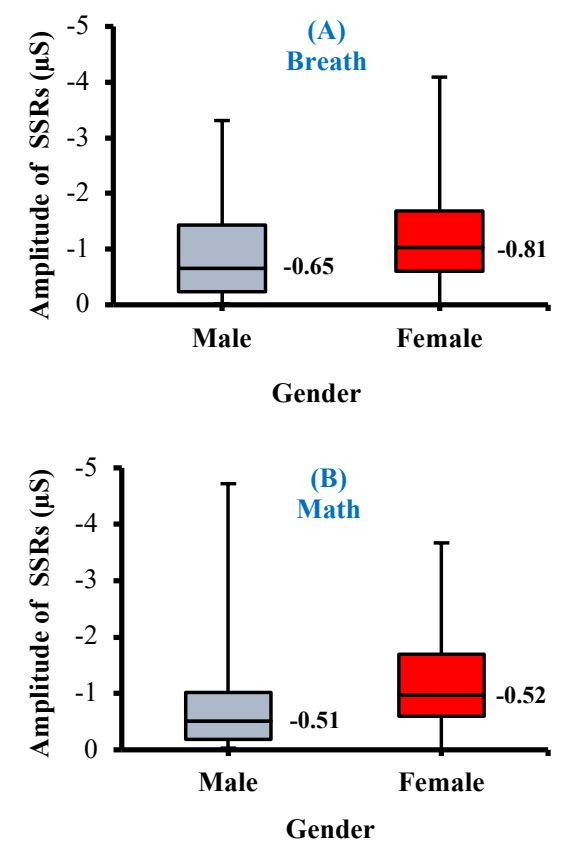

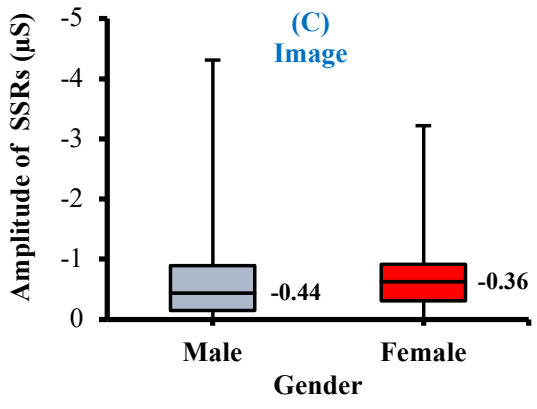

Figure 7 A, B\&C. Box-plot with medians, quartiles, and the minimum and maximum as whiskers shows amplitude of SSRs for both genders as a function of deep breath, math, and image stimuli.

\section{DISCUSSION}

In this study, author identified small sex differences in tonic and phasic EDA parameters to external stimuli. However, almost findings of the study were statistically insignificant $(p>0.05)$ as assessed by Mann-Whitney U test.

Gender differences were present in all three tonic EDA (SCL, SPL and SSL) parameters, but insignificantly $(p>0.05)$, except for SPL, which was significant $(p<0.05)$. The results for SPL are in line with (Aldosky, 2019), who observed a significant difference in SPL with respect to gender differences. Moreover as with (Aldosky, 2019), female showed higher SPL than male (Figure 3). It can be seen in Figure 2 that females exhibited slightly higher SCL than males, which might be due to a greater stress level among females than males. This finding is in line with (Boucsein, 2012; El-Sheikh, 2007), in which women have greater SCL than men. However, it is inconsistent with studies (Eisdorfer et al., 1980; Hare et al., 1971; Neufeld \& Davidson, 1974). A negatively increased (more negative) SPL is found among females than males as seen in Figure 3. The result for the difference between males and females (although not significantly) is in agreement with (Gaviria et al., 1969) who found marked differences between both genders. Also, tonic SS (Figure 4) showed a difference between females and males, which perhaps due to the differences in moisture content or hydration of the corneum among both sexes, since the electrical capacitance of the skin (SS), is proportional to the moisture content or hydration of the corneum (Martinsen et al., 2008). In general, the results for tonic EDA (i.e. SCL, SPL and SSL) are consistent with (Boucsein, 2012) observations based on a thorough literature review in which females had greater tonic EDA than males.

Phasic EDA parameters also showed gender-related differences; however with no statistical significance $(p>0.05)$. Figure $5 \mathrm{~A}$, B\&C illustrates SCRs values for both genders as a function of type of stimulus. It can be seen through the Figure that, females shown larger amplitude of SCRs than males. The finding of a larger SCRs in females than males is supported by the studies of (Bradley et al., 2001; Eisdorfer et al., 1980) and (Venables \& Mitchell, 1996). Also the larger SSRs are obtained with females than males (Figure $7 \mathrm{~A}, \mathrm{~B} \& \mathrm{C}$ ) for all stimuli. These results can be taken in part as supportive of a hypothesis of differential perception of threatening situations by males and females. That the stress (stimuli) response is greater in proportion to the degree of stimuli in females than males is clearly indicated by SCRs and SSRs data. On both measures females exhibit greater response to stimuli (larger amplitude of SCRs and SSRs) than males. In addition, these results have been interpreted as indicating that females generally respond with greater defensive activation than males to stimuli (Kring \& Gordon, 1998). Moreover, phasic (SCRs, SPRs and SSRs) data indicate that females reached their maximal response level under math stimulus, while males responded differentially and showing approximately their highest 
response under image stimulus for the three phasic scores comparing to other stimuli. Gender differences have also been observed with respect to SPRs, but in this case females exhibited lower SPRs (insignificantly) than males as illustrated in Figure 6A, B\&C.

According to (Venables \& Christie, 1973) the observed gender-related differences in EDA recordings can presumably be ascribed to endocrine impacts. Also, gender differences in psychophysiological functioning can be based on the approach of (Broverman et al., 1968). These investigators propose, in part, that a major behaviorallyrelevant physiological difference between males and females lies in differential hormonal characteristics of the two sexes. Particularly, since estrogens are more powerful activating agents than androgens, it can be postulated that females exhibit a higher activation-inhibition balance than do males. This balance should be reflected behaviorally in the predominance of ergotropic over trophotropic behaviors and physiologically in the predominance of sympathetic nervous system activity, males displaying the opposite pattern (Kopacz \& Smith, 1971).

\section{CONCLUSION}

Overall, the study findings suggest that tonic and phasic EDA may constitute gender differences, but with no statistically significance. In addition, females exhibited higher EDA levels and responses as compared with males, except SPRs, which was higher for males. It is probably important to take account of gender or at least record type of gender in carrying out studies using EDA measurements; however the observed gender differences cannot be generalized to clinical applications.

\section{REFERENCES}

Aldosky, H. (2019). Impact of obesity and gender differences on electrodermal activities. General Physiology and Biophysics, 38(6):513 -518.

Bari, D., Aldosky, H., Tronstad, C., Kalvøy, H., \& Martinsen, Ø. (2018). Electrodermal responses to discrete stimuli measured by skin conductance, skin potential, and skin susceptance. Skin Research and Technology, 24(1), 108116.

Boucsein, W. (2012). Electrodermal activity. NewYork, NY: Plenum Press.

Bradley, M. M., Codispoti, M., Sabatinelli, D., \& Lang, P. J. (2001). Emotion and motivation II: sex differences in picture processing. Emotion, 1(3), 300.

Broverman, D. M., Klaiber, E. L., Kobayashi, Y., \& Vogel, W. (1968). Roles of activation and inhibition in sex differences in cognitive abilities. Psychological review, $75(1), 23$.

Edelberg, R. (1971). Electrical properties of the skin. In C.C. Brown (Ed.), Methods in psychophysiology (pp. 1-53). Baltimore: Williams \& Wilkins.

Edelberg, R. (1972). Electrical activity of the skin: Its measurement and uses in psychophysiology. In N. S. Greenfield \& R. A. Sternbach (Eds.), Handbook of psychophysiology (pp. 367-418). New York: Holt, Rinehart, \& Winston.

Eisdorfer, C., Doerr, H. O., \& Follette, W. (1980). Electrodermal reactivity: an analysis by age and sex. Journal of Human Stress, 6(4), 39-42.

El-Sheikh, M. (2007). Children's skin conductance level and reactivity: Are these measures stable over time and across tasks? Developmental Psychobiology, 49(2), 180-186.

Fowles, D. C. (1986). The eccrine system and electrodermal activity. Psychophysiology: Systems, processes, and applications, 1, 51-96.

Fowles, D. C., Christie, M. J., Edelberg, R., Grings, W. W., Lykken, D. T., \& Venables, P. H. (1981). Publication recommendations for electrodermal measurements. Psychophysiology, 18(3), 232-239.
Gaviria, B., Coyne, L., \& Thetford, P. E. (1969). Correlation of skin potential and skin resistance measures. Psychophysiology, $5(5), 465-477$.

Hare, R., Wood, K., Britain, S., \& Frazelle, J. (1971). Autonomic responses to affective visual stimulation: Sex differences. Journal of Experimental Research in Personality.

Jorgenson, R., Salinas, C., Dowben, J., \& St, D. J. (1988). A population study on the density of palmar sweat pores. Birth defects original article series, 24(2), 51-63.

Ketterer, M. W., \& Smith, B. D. (1977). Bilateral electrodermal activity, lateralized cerebral processing and sex. Psychophysiology, 14(6), 513-516.

Kimmel, H., \& Kimmel, E. (1965). Sex differences in adaptation of the GSR under repeated applications of a visual stimulus. Journal of Experimental Psychology, 70(5), 536.

Kopacz, F. M., \& Smith, B. D. (1971). Sex differences in skin conductance measures as a function of shock threat. Psychophysiology, 8(3), 293-303.

Kring, A. M., \& Gordon, A. H. (1998). Sex differences in emotion: expression, experience, and physiology. Journal of personality and social psychology, 74(3), 686.

Maltzman, I., Gould, J., Barnett, O. J., Raskin, D. C., \& Wolff, C. (1979). Habituation of the GSR and digital vasomotor components of the orienting reflex as a consequence of task instructions and sex differences. Physiological Psychology, 7(2), 213-220.

Martinsen, Ø. G., Grimnes, S., Nilsen, J. K., Tronstad, C., Jang, W., Kim, H., Shin, K., Naderi, M., \& Thielmann, F. (2008). Gravimetric method for in vitro calibration of skin hydration measurements. IEEE Transactions on Biomedical Engineering, 55(2), 728-732.

Morimoto, T. (1978). Variations of sweating activity due to sex, age and race. The physiology and pathophysiology of the skin, 5, 16551666.

Neufeld, R. W., \& Davidson, P. O. (1974). Sex differences in stress response: A multivariate analysis. Journal of Abnormal Psychology, 83(2), 178.

Pabst, O., Tronstad, C., Grimnes, S., Fowles, D., \& Martinsen, Ø. G. (2017). Comparison between the AC and DC measurement of electrodermal activity. Psychophysiology, 54(3), 374-385.

Purohit, A. P. (1966). Personality variables, sex-difference, GSR responsiveness and GSR conditioning. Journal of Experimental Research in Personality, 1(3), 166-173.

Tronstad, C., Gjein, G. E., Grimnes, S., Martinsen, Ø. G., Krogstad, A.L., \& Fosse, E. (2008). Electrical measurement of sweat activity. Physiological measurement, 29(6), S407.

Tronstad, C., Grimnes, S., Martinsen, Ø. G., Amundsen, V., \& Wojniusz, S. (2010). PC-based instrumentation for electrodermal activity measurement. Paper presented at the Journal of Physics: Conference Series.

Tronstad, C., Johnsen, G. K., Grimnes, S., \& Martinsen, Ø. G. (2010). A study on electrode gels for skin conductance measurements. Physiological measurement, 31(10), 1395.

Tronstad, C., Kalvøy, H., Grimnes, S., \& Martinsen, Ø. G. (2013). Improved estimation of sweating based on electrical properties of skin. Annals of biomedical engineering, 41(5), 1074-1083.

Venables, P., \& Christie, M. (1973). Mechanisms, instrumentation, recording techniques, and quantification of responses. In W. F. Prokasy \& D. C. Raskin (Eds.), Electrodermal activity in psychological research (pp. 1-124). New York: Academic.

Venables, P., \& Mitchell, D. (1996). The effects of age, sex and time of testing on skin conductance activity. Biological psychology, $43(2), 87-101$. 